\title{
A Coh-Metrix Analysis of Two Textbooks: Successful English for Vocational Colleges and Vocational College English (An Integrated Skills Course)
}

\author{
ZHANG Rong-gen \\ Shanghai Publishing and Printing College, Shanghai, China
}

\begin{abstract}
This paper, by making a comparative Coh-Metrix analysis of two Vocational Colleges English textbooks, hopes to give some enlightenment on vocational college English teaching in China. The findings are that with smaller numbers of sentences in simpler structure and full of more familiar content words, the texts are easier to read, while with larger numbers of words, especially complex modifying noun phrases, in greater lexical diversity, the texts are more difficult to read. The final suggestion is that both textbooks can be used in graded English teaching atmosphere.
\end{abstract}

Keywords: Coh-Metrix, Vocational Colleges English, textbook

\section{Introduction}

Coh-Metrix is a computational tool that produces indices of the linguistic and discourse representations of a text. Coh-Metrix was primarily used to improve reading comprehension in classrooms. Then it is used to calculate the coherence texts on many different measures and to study vocabulary (Graesser et al., 2004, 2011, 2013). Researches on text via Coh-Metrix are mainly as follows:

Carroll (2007) examines the initial and final versions of a term paper, indicating that students express themselves with less tentativeness at the end of the semester, and are consistent with the suggestion that students move from relativism to commitment during the course of a semester, and that the LIWC (Linguistic Inquiry and Word Count) may detect differences between different writing genres. Wang and Cho (2010) examines argumentative and technical writings by undergraduate student-produced texts and finds students using genre-dependent cohesive devices can inform the instructional librarians in a limited way to write research papers.

GUI (2010) investigates lexical meanings and meaning relations in L1 and L2 writings at the deep structure level via Coh-Metrix, finding that L2 writings use more known information to establish lexical co-referentiality, while L1 writings manifest more lexical variety and sophistication.

YANG (2011) makes a comparative study of the reading comprehension texts in China's Matriculation English Test, Tests for English Majors (TEM-4) and (TEM-8), and finds text readabilities in narrative, expository,

ZHANG Rong-gen, associate professor, M.A., Basic Science Education Department, Shanghai Publishing and Printing College. 
and argumentative texts differing significantly and increasingly.

LI (2012) explores thesis genre through text and context, and its significance in three aspects: being problem-oriented, taking thesis writing as both social and discursive from textual, contextual, and critical perspectives, and focusing on Chinese postgraduate English thesis.

HE (2013) conducts a comparative analysis on reading texts of TEM8 and IELTS from the perspective of text complexity, and finds (1) Texts in TEM8 have a wider lexical coverage of high frequency words while lexical coverage of low frequency words in texts of IELTS is denser than that of TEM8 according to word frequency distribution of the two tests; texts of IELTS is longer than TEM8 counterpart and more diverse words and more concrete words are employed in texts of TEM8; (2) Syntactical structure in TEM8 is more complicated than that in IELTS; and (3) TEM8 tend to use referential cohesion whereas texts in IELTS are testified to be better in deep cohesion and LSA (Latent Semantic Analysis) overlap between paragraphs.

Serrano (2014) studies the dynamic effects that a read text causes on the working memory of readers by means of a connectionist model of memory during reading, and analyzes the correlation of those effects with the essay quality scores and text linguistic features, proving how BICA (Biologically Inspired Cognitive Architectures) models can feedback Neuroscience and Psychology.

The above literature review shows that researches via Coh-Metrix are mostly on university undergraduates and postgraduates, while such researches are rarely found to have been done on vocational college students. Therefore, this paper attempts to fill in such a gap by making a comparative Coh-Metrix analysis of two textbooks: Successful English for Vocational Colleges (SE for short below) and Vocational College English (An Integrated Skills Course, VCE for short) (ZHUANG, 2014; WANG, 2008), in hope to give some enlightenment on vocational college English teaching in China.

\section{Methodology}

Twenty-eight texts are processed respectively from the above textbooks by SPSS and Coh-Metrix 3.0.

Concepts concerned in data processing:

DESPC - Paragraph count, number of paragraphs;

DESSC - Sentence count, number of sentences;

DESWC - Word count, number of words;

DESSL - Sentence length, number of words, mean;

PCNARp - Text Easability PC Narrativity, percentile;

PCSYNp-Text Easability PC Syntactic simplicity, percentile;

PCCNCp - Text Easability PC Word concreteness, percentile;

CRFAOa - Argument overlap, all sentences, binary, mean;

CRFCWO1- Content word overlap, adjacent sentences, proportional, mean;

LDTTRc - Lexical diversity, type-token ratio, content word;

LDTTRa - Lexical diversity, type-token ratio, all words;

SMTEMP—Temporal cohesion, tense and aspect repetition, mean;

SYNNP - Number of modifiers per noun phrase, mean;

SYNSTRUTt - Sentence syntax similarity, all combinations, across paragraphs, mean; 
WRDFAMc - Familiarity for content words, mean;

WRDCNCc - Concreteness for content words, mean;

WRDHYPn — Hypernymy for nouns, mean;

WRDHYPv—Hypernymy for verbs, mean;

RDFRE-Flesch Reading Ease;

RDFKGL-Flesch-Kincaid Grade Level;

RDL2-Coh-Metrix L2 Readability.

\section{Results and Analysis}

Table 1

Descriptive Statistics

\begin{tabular}{lllllllll}
\hline & VCE Min & SE Min & VCE Max & SE Max & VCE mean & SE mean & VCE Std. & SE Std. \\
\hline DESPC & 9.00 & 8.00 & 34.00 & 48.00 & 20.67 & 20.96 & 6.70 & 10.66 \\
DESSC & 21.00 & 17.00 & 74.00 & 140.00 & 40.38 & 55.00 & 11.54 & 26.93 \\
DESWC & 289.00 & 294.00 & 747.00 & 1470.00 & 477.00 & 704.29 & 134.15 & 298.18 \\
DESSL & 8.25 & 8.33 & 18.68 & 18.97 & 12.11 & 13.32 & 2.76 & 2.74 \\
PCNARp & 26.43 & 15.39 & 97.26 & 85.99 & 69.46 & 52.13 & 23.92 & 21.63 \\
PCSYNp & 40.90 & 35.57 & 86.86 & 92.36 & 70.97 & 68.51 & 12.22 & 12.04 \\
PCCNCp & 16.11 & 12.71 & 99.46 & 77.64 & 58.98 & 38.95 & 25.02 & 20.08 \\
CRFAOa & 0.13 & 0.15 & 0.53 & 0.63 & 0.34 & 0.30 & 0.11 & 0.13 \\
CRFCWO1 & 0.03 & 0.04 & 0.18 & 0.21 & 0.09 & 0.09 & 0.03 & 0.05 \\
LDTTRc & 0.51 & 0.24 & 0.79 & 0.76 & 0.67 & 0.64 & 0.08 & 0.11 \\
LDTTRa & 0.34 & 0.15 & 0.57 & 0.56 & 0.46 & 0.44 & 0.06 & 0.08 \\
SMTEMP & 0.70 & 0.68 & 0.98 & 0.98 & 0.86 & 0.87 & 0.07 & 0.07 \\
SYNNP & 0.47 & 0.49 & 1.05 & 0.98 & 0.73 & 0.80 & 0.18 & 0.13 \\
SYNSTRUTt & 0.05 & 0.06 & 0.18 & 0.14 & 0.10 & 0.08 & 0.03 & 0.02 \\
WRDFAMc & 573.45 & 563.18 & 593.01 & 592.56 & 582.66 & 579.33 & 4.88 & 6.19 \\
WRDCNCc & 353.58 & 337.23 & 446.07 & 403.42 & 385.24 & 370.54 & 21.88 & 17.65 \\
WRDHYPn & 5.39 & 5.38 & 7.25 & 7.25 & 6.45 & 6.37 & 0.49 & 0.56 \\
WRDHYPv & 1.31 & 1.31 & 1.85 & 1.94 & 1.56 & 1.58 & 0.15 & 0.15 \\
RDFRE & 62.33 & 54.42 & 96.14 & 89.09 & 79.47 & 69.65 & 8.40 & 9.09 \\
RDFKGL & 2.28 & 2.98 & 8.19 & 10.26 & 5.18 & 6.85 & 1.58 & 1.77 \\
RDL2 & 11.76 & 9.90 & 27.35 & 30.34 & 18.87 & 18.27 & 4.32 & 4.91
\end{tabular}

Table 2

Difference of the Basic Textual Features

\begin{tabular}{lllllllll}
\hline & DESSC & DESWC & PCNARp & PCCNCp & WRDFAMc & WRDCNCc & RDFRE & RDFKGL \\
$\mathrm{t}$ & -2.445 & -3.406 & 0.2632 & 3.06 & 2.068 & 2.562 & 3.886 & -3.451 \\
$\mathrm{p}$ & 0.018 & 0.001 & 0.012 & 0.004 & 0.044 & 0.014 & 0 & 0.001 \\
\hline
\end{tabular}

From Table 1 and Table 2, the results are shown as follows:

The first measurement of significant difference between SE and VCE is DESSC, the number of sentences in the corresponding texts, with independent samples t-test $p=0.018<0.05$, and with the mean of 55.00 and 40.38 respectively. The second measurement of significant difference between SE and VCE is DESWC, the number of words in the corresponding texts, with independent samples t-test $\mathrm{p}=0.001<0.01$, and with the mean of 298.18 
and 134.15 respectively. Both of these prove each text in SE is relatively longer than that in VCE.

The third measurement of significant difference between SE and VCE is PCNARp, the percentile of Text Easability and Narrativity, in the corresponding texts, with independent samples t-test $\mathrm{p}=0.012<0.05$, and with the mean of 52.13 and 69.46 respectively. The fourth measurement of significant difference between SE and VCE is PCCNCp, the percentile of Text Easability and Word concreteness, in the corresponding texts, with independent samples t-test $p=0.004<0.05$, and with the mean of 38.95 and 58.98 resepectively. Both of these shows each text in VCE is relatively easier to read and more narrative than that in SE.

The fifth measurement of significant difference between SE and VCE is WRDFAMc, familiarity for content words, in the corresponding texts, with independent samples t-test $\mathrm{p}=0.044<0.05$, and with the mean of 579.33 and 582.66 resepectively. The sixth measurement of significant difference between SE and VCE is WRDCNCc, Concreteness for content words in the corresponding texts, with independent samples t-test $\mathrm{p}=0.014<0.05$, and with the mean of 370.54 and 385.24 resepectively. Both of the fifth and sixth measure show that texts in VCE have more familiar concrete content words than those in SE. This reconfirms the previous result that both of these show each text in VCE is relatively easier to read and more narrative than that in SE.

The seventh measurement of significant difference between SE and VCE is RDFRE, Flesch Reading Ease in the corresponding texts, with independent samples t-test $\mathrm{p}=0<0.01$, and with the mean of 69.65 and 79.47 resepectively. The eighth measurement of significant difference between SE and VCE is RDFKGL, Flesch-Kincaid Grade Level in the corresponding texts, with independent samples t-test $p=0.001<0.01$, and with the mean of 6.85 and 5.18 resepectively. These last two measurements further confirm that the texts in SE are relatively more difficult to understand than those in VCE.

Table 3

Textbook Pearson Correlations

\begin{tabular}{llllllllll}
\hline DESPC & DESSC & DESWC & DESSL & PCNARp & PCSYNp & PCCNCp & CRFAOa & CRFCWO1 LDTTRc \\
\hline 0.017 & $0.339^{*}$ & $0.449^{* *}$ & 0.22 & $-0.362^{*}$ & -0.10 & $-0.411^{* *}$ & -0.20 & -0.08 & -0.20 \\
\hline LDTTRa SMTEMP & SYNNP & SYNSTRUTt & WRDFAMc & WRDCNCc & WRDHYPn & WRDHYPv & RDFRE & RDFKGL \\
\hline-0.16 & 0.02 & 0.25 & -0.26 & $-0.292^{*}$ & $-0.353^{*}$ & -0.08 & 0.06 & $-0.497^{* *}$ & $0.454^{* *}$ \\
\hline
\end{tabular}
Textbook; 2- SE Textbook.

Table 3 shows there are three significant correlations in SE as following: DESSC/0.339*, DESWC/0.449**, RDFKGL/0.454**. On the other hand, there are four significant correlations in VCE as following: PCNARp/-0.362*, PCCNCp/-0.411**, WRDFAMc/-0.292*, WRDCNCc/-0.353*, RDFRE/-0.497**. Hence, the above significantly correlated indices are detailed in the following section.

\section{Discussion and Conclusion}

Correlations of indices with DESSC, DESWC, and RDFKGL in SE are found in data processing as follows: First, there are four correlations concerning DESSC: DESSC/DESPC $\left(0.859^{* *}\right)$, CRFCWO1/DESSC $\left(-0.407^{*}\right)$, LDTTRc/DESSC $\left(-0.729^{* *}\right)$, and RDL2/DESSC $\left(-0.451^{*}\right)$. DESSC is strongly correlated with DESPC, i.e., in the texts of SE, the number of paragraphs is proportional to the number of sentences. Nevertheless, DESSC is negatively correlated with CRFCWO1, i.e., content word overlap appears less often with the 
increasing number of sentences. Besides, DESSC is negatively correlated with LDTTRc and RDL2, i.e., lexical diversity and text Readability become lower with the increasing number of sentences.

Second, there are three correlations concerning DESWC: DESWC/DESPC $\left(0.770^{* *}\right)$, LDTTRc/DESWC $\left(-0.519^{* *}\right)$, and RDL2/DESWC $\left(-0.540^{* *}\right)$. DESWC is strongly correlated with DESPC, i.e., the number of words is proportional to the number of paragraphs. Whereas, DESWC is negatively correlated with LDTTRc and RDL2, i.e., lexical diversity and text readability become lower with the increasing number of sentences.

Third, there are seven correlations concerning RDFKGL: RDFKGL/DESSL $\left(0.820^{* *}\right)$, RDFKGL/LDTTRa $\left(0.456^{*}\right)$, RDFKGL/SYNNP (0.531**), RDFKGL/PCNARp (-0.639**), RDFKGL/PCSYNp (-0.486*), RDFKGL/WRDFAMc $\left(-0.516^{* *}\right)$, and RDFKGL/RDFRE $\left(-0.952^{* *}\right)$. RDFKGL is strongly correlated with DESSL, LDTTRa, and SYNNP, i.e., Flesch-Kincaid Grade Level of the text is proportional to the number of words, lexical diversity, and the number of modifiers per noun phrase; while RDFKGL is negatively correlated with PCNARp, PCSYNp, WRDFAMc, and RDFRE, i.e., Flesch-Kincaid Grade Level of the text becomes lower with the increase of Syntactic simplicity, Familiarity for content words, and Flesch Reading Ease.

To summarize this subsection, the three above results show, with less numbers of sentences in simpler structure and full of more familiar content words, VCE texts are easier to read, but there are more SE texts filled with larger numbers of words, especially complex modifying noun phrases, in greater lexical diversity, causing them more difficult to read.

Correlations of indices with PCNARp, PCCNCp, WRDFAMc, WRDCNCc, and RDFRE inVCE are found in data processing as follows:

First, there are five correlations concerning PCNARp: SYNNP/PCNARp $\left(-0.857^{* *}\right)$, PCNARp/RDFRE (-0.505*), WRDHYPv/PCNARp $(0.481 *)$, PCNARp/RDFRE $\left(0.658^{* *}\right)$, and PCNARp/RDL2 $\left(0.690^{* *}\right)$. PCNARp is negatively correlated with SYNNP and RDFRE, i.e., with greater number of modifiers per noun phrase, the text is less narrative and more difficult to read; while PCNARp is strongly correlated with WRDHYPv, RDFRE, and RDL2, i.e., texts with more hypernymous verbs are easier to read.

Second, Although WRDFAMc negatively correlated with PCSYNp $\left(-0.532^{* *}\right)$, i.e., texts in VCE are filled with familiar content words, but in less simple syntactic structure; WRDCNCc is strongly correlated with PCCNCp $\left(0.870^{* *}\right)$, i.e., the texts are filled with more concrete content words. Hence such texts are easier to read.

Third, there are five correlations concerning RDFRE: DESWC/RDFRE $\left(0.558^{* *}\right)$, PCSYNp/RDFRE $\left(0.741^{* *}\right)$, SMTEMP/RDFRE $\left(0.441^{*}\right)$, WRDHYPv/RDFRE $(0.463 *)$, SYNNP/RDFRE $(-0.442 *)$, and RDFRE /RDFKGL $\left(-0.920^{* *}\right)$. RDFRE is strongly correlated with DESWC, PCSYNp, and SMTEMP, i.e., although the texts in VCE are filled with quite a number of words, yet their syntactic structures are simple, with various repetitions of tense and aspect. RDFRE is negatively correlated with SYNNP and RDFKGL, i.e., some texts with a great number of modifiers per noun phrase, yet their Flesch-Kincaid Grade Level is low. Here, once more, it confirms texts in VCE are easier to read.

To summarize the above discussions, in view of the Flesch-Kincaid Grade Level, both textbooks are lower than American high school Grade 7 students, which means neither of them (VCE 5.18, vs. SE 6.85) can meet US college English level. ${ }^{1}$ Despite this, since the English proficiency of the students in China seems to be lowering

\footnotetext{
${ }^{1}$ See http://www.zybang.com/question/45eff373e1aba376f667eefdfcd89aa6.html.
} 
in recent years (LI, 2012), that of vocational students is also no exception. VCE textbook has been used in my college since 2009. The overall feedback of most students is that it is a bit simple, except that few students think it a little difficult, which may result from their weak motivation to study to some degree. Therefore, my suggestion is that in graded English teaching atmosphere, mostly SE can be used as a textbook by our vocational college students of higher level, whereas VCE can be used by those of lower English level. Of course, since SE was just published in 2014, its practicality in graded English teaching of vocational college students still waits to be confirmed.

\section{References}

Carroll, D. W. (2007). Patterns of student writing in a critical thinking course: A quantitative analysis. Assessing Writing, 12(1), 213-227.

Graesser, A. C., Dowell, N., \& Moldovan, C. (2011). A computers understanding of literature. Scientific Study of Literature, 1(1), 24-33. doi:10.1075/ssol.1.1.03gra

Graesser, A. C., McNamara, D. S., \& Kulikowich, J. M. (2011). Coh-Metrix: Providing multilevel analyses of text characteristics. Educational Researcher, 40(5), 223-234.

Graesser, A. C., McNamara, D. S., Louwerse, M. M., \& Cai, Z. (2004). Coh-metrix: Analysis of text on cohesion and language. Behavior Research Methods, Instruments, \& Computers: A Journal of the Psychonomic Society, 36(2), 193-202.

GUI, L. (2010). A contrastive study of lexical proficiency between L1 and L2 compositions via computerized assessment. Foreign Language Teaching and Research, 42(6), 445-450.

HE, L. Q. (2013). A comparative study on text complexity of reading comprehension in TEM8 and IELTS based on Coh-Metrix (M.A. thesis, College of International Studies of Southwest University, Chongqing).

LI, X. K. (2012). Exploring the generic features of M.A. theses in linguistics (Ph.D. thesis, Zhejiang University, Zhejiang).

LI, Y. J. (2012). Negative echoic effects of the writing topic of college English test band 4-Some afterthought of a CET4 writing rater. College English Academic Edition, 99(1), 273-277.

McNamara, D. S., Graesser, A. C., McCarthy, P. M., \& Cai, Z. (in press). Automated evaluation of text and discourse withCoh-Metrix. Cambridge, M.A.: Cambridge University Press.

McNamara, D. S., Louwerse, M. M., Cai, Z., \& Graesser, A. (2013). Coh-Metrix version 3.0 [Software]. Retrieved February 3, 2016 from http//:cohmetrix.com

Serrano, J. I, Castillo, M. D, \& Iglesias, Á. (2014). Effects of text essay quality on readers' working memory by a computational model. Biologically Inspired Cognitive Architectures, 7(1), 39-52.

WANG, S. (2008). Vocational college English (An integrated skills course). Shanghai: Shanghai Foreign Language Education Press.

YANG, P. (2011). An empirical study of the influence of style on the readability of English text. Overseas English, 3, $214-220$.

ZHUANG, Z. X. (2014). Successful English for vocational colleges. Shanghai: Shanghai Foreign Language Education Press. 